\title{
Architecture for a large-scale ion-trap quantum computer
}

\author{
D. Kielpinski, C. Monroe $\dagger$ \& D. J. Wineland $\ddagger$ \\ * Research Laboratory of Electronics and Center for Ultracold Atoms, Massachusetts Institute of Technology, Cambridge, Massachusetts 02139, USA \\ $\dagger$ FOCUS Center and Department of Physics, University of Michigan, Ann Arbor, Michigan 48109-1120, USA \\ $\ddagger$ Time and Frequency Division, National Institute of Standards and Technology, Boulder, Colorado 80305, USA
}

Among the numerous types of architecture being explored for quantum computers are systems utilizing ion traps, in which quantum bits (qubits) are formed from the electronic states of trapped ions and coupled through the Coulomb interaction. Although the elementary requirements for quantum computation have been demonstrated in this system, there exist theoretical and technical obstacles to scaling up the approach to large numbers of qubits. Therefore, recent efforts have been concentrated on using quantum communication to link a number of small ion-trap quantum systems. Developing the array-based approach, we show how to achieve massively parallel gate operation in a large-scale quantum computer, based on techniques already demonstrated for manipulating small quantum registers. The use of decoherence-free subspaces significantly reduces decoherence during ion transport, and removes the requirement of clock synchronization between the interaction regions.

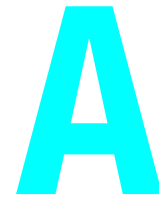

quantum computer is a device that prepares and manipulates quantum states in a controlled way, offering significant advantages over classical computers in tasks such as factoring large numbers ${ }^{1}$ and searching large databases ${ }^{2}$. The power of quantum computing derives from its scaling properties: as the size of these problems grows, the resources required to solve them grow in a manageable way. Hence a useful quantum computing technology must allow control of large quantum systems, composed of thousands or millions of qubits.

The first proposal for ion-trap quantum computation involved confining a string of ions in a single trap, using their electronic states as qubit logic levels, and transferring quantum information between ions through their mutual Coulomb interaction ${ }^{3}$. All the elementary requirements for quantum computation ${ }^{4}$-including efficient quantum state preparation ${ }^{5-7}$, manipulation ${ }^{7-10}$ and read-out ${ }^{7,11,12}$-have been demonstrated in this system. But manipulating a large number of ions in a single trap presents immense technical difficulties, and scaling arguments suggest that this scheme is limited to computations on tens of ions ${ }^{13-15}$. One way to escape this limitation involves quantum communication between a number of small ion-trap quantum registers. Recent proposals along these lines that use photon coupling ${ }^{16-18}$ and spin-dependent Coulomb interactions ${ }^{19}$ have not yet been tested in the laboratory. The scheme presented here, however, uses only quantum manipulation techniques that have already been individually experimentally demonstrated.

\section{The quantum CCD}

To build up a large-scale quantum computer, we have proposed a 'quantum charge-coupled device' (QCCD) architecture consisting of a large number of interconnected ion traps. By changing the operating voltages of these traps, we can confine a few ions in each trap or shuttle ions from trap to trap. In any particular trap, we can manipulate a few ions using the methods already demonstrated, while the connections between traps allow communication between sets of ions ${ }^{13}$. Because both the speed of quantum logic gates ${ }^{20}$ and the shuttling speed are limited by the trap strength, shuttling ions between memory and interaction regions should consume an acceptably small fraction of a clock cycle.

Figure 1 shows a diagram of the proposed device. Trapped ions storing quantum information are held in the memory region. To perform a logic gate, we move the relevant ions into an interaction region by applying appropriate voltages to the electrode segments. In the interaction region, the ions are held close together, enabling the Coulomb coupling necessary for entangling gates ${ }^{3,21}$. Lasers are focused through the interaction region to drive gates. We then move the ions again to prepare for the next operation.

We can realize the trapping and transport potentials needed for the QCCD using a combination of radio-frequency (r.f.) and quasistatic electric fields. Figure 1 shows only the electrodes that support the quasistatic fields. By varying the voltages on these electrodes, we confine the ions in a particular region or transport them along the local trap axis, which lies along the thin arrows in Fig. 1. Two more layers of electrodes lie above and below the static electrodes, as shown in Fig. 2. Applying r.f. voltage to the outer layers creates a quadrupole field that confines the ions transverse to the local trap axis by means of the ponderomotive force ${ }^{22}$. This geometry allows stable transport of the ions around ' $T$ ' and ' $\mathrm{X}$ ' junctions, so we can build complex, multiply connected trap structures.

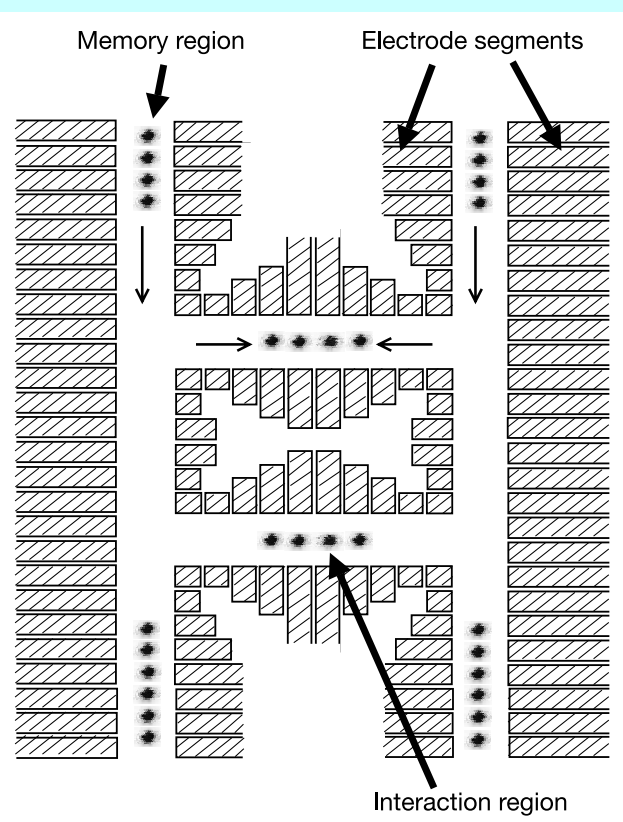

Figure 1 Diagram of the quantum charge-coupled device (QCCD). Ions are stored in the memory region and moved to the interaction region for logic operations. Thin arrows show transport and confinement along the local trap axis. 
Electrode structures for the QCCD are relatively easy to fabricate. A number of ion traps have been built by laser-machining slits in alumina wafers and evaporating gold electrodes onto the alumina ${ }^{23}$. These traps have geometries similar to that needed for the QCCD, and have spacings between the static and r.f. electrodes of fractions of a millimetre, allowing confinement frequencies up to $20 \mathrm{MHz}$ for r.f. field frequencies of $\sim 250 \mathrm{MHz}$. Similar electrode structures are currently being constructed from heavily doped silicon using standard microfabrication techniques ${ }^{24}$. Here the silicon acts as the conductive electrode material, while glass spacers anodically bonded to the silicon insulate the r.f. electrodes from the static electrodes.

A first step towards a QCCD has been taken at the National Institute of Standards and Technology (NIST) by constructing a pair of interconnected ion traps; the individual traps are similar to those used in previous work ${ }^{23}$ and are separated by $1.2 \mathrm{~mm}$. Efficient coherent transport of a qubit between the two traps was demonstrated by performing a Ramsey-type experiment involving the two traps, where no loss of contrast within the experimental error $(\sim 0.6 \%)$ was observed ${ }^{25}$. Transport times were as short as $\sim 50 \mu \mathrm{s}$, with corresponding ion velocities greater than $10 \mathrm{~m} \mathrm{~s}^{-1}$ (see also ref. 26). The transport did not cause any heating of the ion motion or shortening of ion lifetime in the trap.

To maximize the clock speed of the QCCD, we need to transport ions quickly. However, the entangling gate demonstrated in previous work at $\mathrm{NIST}^{9,21}$ has low error only for ions cooled near the quantum ground state. To recool the ions after transport and to counteract the effects of heating ${ }^{23}$, we propose to use sympathetic cooling of the ions used for quantum logic by another ion species $^{13,27,28}$. Confining both species in the interaction region lets us use the cooling species as a heat sink, with the Coulomb interaction transferring energy between the two species, as experimentally demonstrated in refs 29-31.

\section{Decoherence}

Whereas the decoherence and gate errors in single-trap quantum registers have already been characterized, additional decoherence can occur during ion transport. For instance, the energy splitting of the qubit states of an ion depends on the magnetic field at the ion through the Zeeman effect. During ion transport, the spatial variations of the magnetic field strength along the transport path cause the qubit states to acquire a path-dependent relative phase $\alpha$, so that, for example, $|\downarrow\rangle+|\uparrow\rangle \rightarrow|\downarrow\rangle+\mathrm{e}^{i \alpha}|\uparrow\rangle$ over transport. If we do not know $\alpha$, we have lost the phase information, effectively dephasing the quantum state. But knowing $\alpha$ for all relevant paths is tantamount to characterizing the magnetic field on micrometre

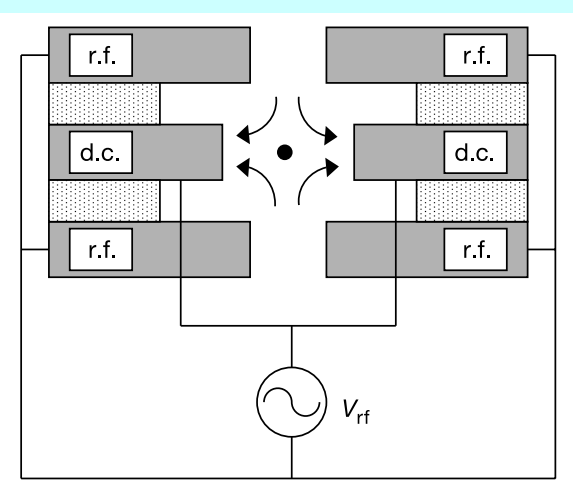

Figure 2 Configuration of radio-frequency (r.f.) and static (d.c.) electrodes for the QCCD. Dotted regions indicate insulating spacers. Applying high r.f. voltage to the two outer electrode layers while keeping the inner layer at r.f. ground creates the r.f. quadrupole field shown by the arrows. This field provides trapping potentials for confinement transverse to the local trap axis, which points perpendicular to the page and is located at the central black dot. View in Fig. 1 is from the top of this figure. length scales across the entire device, a very difficult task.

Retaining phase information during a computation also requires accurate positioning of the ions in the interaction regions. As the logic gate parameters depend on the phase of the driving laser fields at the ion positions, the ion positions and laser path lengths must be controllable with accuracies much better than an optical wavelength. Although this does not place unreasonable constraints on the accuracy of the voltage sources driving the QCCD electrodes, stray electric fields emanating from the electrodes or mechanical vibration of the QCCD can readily move the ions a fraction of a wavelength from their nominal positions, effectively adding a relative phase $\alpha$ of the type considered above.

\section{Decoherence-free encoding}

We can reduce these sources of decoherence by several orders of magnitude by encoding each qubit into a decoherence-free subspace (DFS) of two ions ${ }^{32-34}$. This DFS is spanned by the states $|0\rangle=|\downarrow \uparrow\rangle$, $|1\rangle=|\uparrow \downarrow\rangle$ of the ions. We refer to the ions as 'physical qubits' and call the effective two-level system formed by $|0\rangle,|1\rangle$ a 'logical qubit'. If the state $|\uparrow\rangle$ of ion $j$ acquires a phase $\alpha_{j}$ over and above the predicted phase $\alpha_{0}$ for the transport path, we see

$$
|0\rangle+|1\rangle \rightarrow \mathrm{e}^{i \alpha_{1}}|\downarrow \uparrow\rangle+\mathrm{e}^{i \alpha_{2}}|\uparrow \downarrow\rangle=|0\rangle+\mathrm{e}^{i \Delta \alpha}|1\rangle
$$

where we write $\Delta \alpha \equiv \alpha_{2}-\alpha_{1}$. If $\Delta \alpha=0$, we see that the DFS logical qubit is unaffected by the acquired phase. Here the phases $\alpha_{i}$ are themselves unknown, but the ions acquire the same unknown phase, a process called collective dephasing. The logical qubit decoheres only insofar as the dephasing fails to be collective.

As an example, assume that the physical qubit energies have a linear dependence on magnetic field and that the field varies linearly over an extended QCCD device of size $10 \mathrm{~cm}$. If each pair of physical qubits comprising a logical qubit is separated on average by $10 \mu \mathrm{m}$, a logical qubit dephases more slowly than a physical qubit by a factor of $10^{4}$. Again, Stark shifts of the qubit levels can be induced by the electric fields that push the ions from place to place, but their dephasing effects on the logical qubits are also suppressed. In general, the effect of any external field varying over a length scale $L_{\text {ext }}$ and inducing energy shifts of $p$ th power in the field is suppressed by a factor $\left(L_{\text {ext }} / d\right)^{p}$ for DFS encoding. A DFS-encoded qubit is therefore robust against decoherence incurred during transport.

We can also perform universal quantum logic in the DFS, as we now show. If we hold two ions in an interaction region, we can use the entangling gate of refs 9 and 21 to apply the operator

$$
\begin{aligned}
\mathrm{U}_{2}\left(\theta, \phi_{1}, \phi_{2}\right) & =\cos \theta\left[\mathrm{I}_{1} \otimes \mathrm{I}_{2}\right]+i \sin \theta\left[\mathrm{X}_{\phi_{1}} \otimes \mathrm{X}_{\phi_{2}}\right] \\
& =\cos \theta \mathrm{I}^{\mathrm{DFS}}+i \sin \theta \mathrm{X}_{\Delta \phi_{12}}^{\mathrm{DFS}} \\
\mathrm{X}_{\phi} & \equiv \mathrm{X} \cos \phi+\mathrm{Y} \sin \phi
\end{aligned}
$$

where $\mathrm{X}, \mathrm{Y}$ are the Pauli operators and the superscript 'DFS' indicates that the operator acts in the DFS logical basis. Though the individual phases $\phi_{1}, \phi_{2}$ depend sensitively on the path-length differences of the driving lasers over the macroscopic distance from the lasers to the ions ${ }^{13}$, the DFS gate phase $\Delta \phi_{12}=\phi_{1}-\phi_{2}$ depends only on the microscopic path length of the driving laser between the two ions, which can be readily controlled by small adjustments of the trap voltages ${ }^{9,12,24}$. The DFS encoding makes the computation insensitive to spatial phase fluctuations; as we will see, it protects against temporal phase fluctuations as well. We can set $\theta$ over a wide range of values ${ }^{21,24}$, so the two-ion entangling gate lets us perform arbitrary rotations of a single logical qubit. Using the same entangling gate on four ions, we can obtain the operator

$$
\begin{gathered}
\mathrm{U}_{4}=\frac{1}{\sqrt{2}}\left[\mathrm{I}_{1} \otimes \mathrm{I}_{2} \otimes \mathrm{I}_{3} \otimes \mathrm{I}_{4}-i \mathrm{X}_{\phi_{1}} \otimes \mathrm{X}_{\phi_{2}} \otimes \mathrm{X}_{\phi_{3}} \otimes \mathrm{X}_{\phi_{4}}\right] \\
=\frac{1}{\sqrt{2}}\left[\mathrm{I}^{\mathrm{DFS}} \otimes \mathrm{I}^{\mathrm{DFS}}-i \mathrm{X}_{\Delta \phi_{12}}^{\mathrm{DFS}} \otimes \mathrm{X}_{\Delta \phi_{34}}^{\mathrm{DFS}}\right]
\end{gathered}
$$


where ions 1 and 2 encode one logical qubit and ions 3 and 4 encode another, and we write $\Delta \phi_{34}=\phi_{3}-\phi_{4}$. This operator is equivalent to an XOR in the logical basis up to rotations of single logical qubits $^{35}$, so the operators of equations (2) and (5) suffice for universal quantum logic.

To use the DFS encoding in a large-scale quantum computation, we initialize the ions in pairs to the state $|\downarrow \uparrow\rangle$. Each pair of ions remains in the DFS through the quantum computation, so the logical qubits resist transport decoherence and all other types of collective dephasing. Read-out of the DFS qubit is straightforward, as we need only discriminate between $|\downarrow \uparrow\rangle$ and $|\uparrow \downarrow\rangle$. All the operators needed for universal quantum logic in the DFS have already been experimentally implemented ${ }^{9,24}$, so we should be able to use the DFS encoding in a large-scale quantum computer. Notably, all logic gate operations can be accomplished by uniformly illuminating the ions in the interaction region, removing the need for tightly focused laser beams.

\section{Logic gate synchronization}

The DFS encoding also removes the requirement of clock synchronization between logic gates, a major but little-recognized obstacle to large-scale parallel quantum computation. As the energy levels of our physical qubits are non-degenerate, we must keep track of the resulting phase accumulation to preserve the quantum information in the physical qubit basis ${ }^{34}$. Parallel operations taking place in many interaction regions thus require clocks that remain synchronized over the whole computation time ${ }^{36}$. Synchronization can become very difficult for many qubits: for a transition frequency $\omega_{0}$ between $|\downarrow\rangle$ and $|\uparrow\rangle$, the two components of the state $|\downarrow\rangle^{N}+|\uparrow\rangle^{N}$ acquire a significant relative phase in a time $\sim 1$ / $\left(N \omega_{0}\right)$. To maintain phase stability of the computation, we therefore require a frequency reference with fractional frequency stability much better than $\lesssim 1 /\left(N \omega_{0} \tau\right)$ at an averaging time $\tau$ equal to the duration of the quantum computation.

To be concrete, we consider trapped ${ }^{40} \mathrm{Ca}^{+}$ions as qubits, with the ground $\mathrm{S}_{1 / 2}$ state and metastable $\mathrm{D}_{5 / 2}$ states as logic levels. This system is being investigated for quantum computation by a number of groups $s^{7,15,37}$. Here the transition frequency is $412 \mathrm{THz}$, comparable to the $533 \mathrm{THz}$ operating frequency of the currently most stable laser oscillator ${ }^{38}$, which has a fractional frequency instability of $3 \times 10^{-16}$ as $1 \mathrm{~s}$ averaging time. If the computation takes about $1 \mathrm{~s}$, equal to the lifetime of the metastable $\mathrm{D}_{5 / 2}$ state, we see that current technology barely provides the appropriate phase stability for even one ion. Of course, this argument can be regarded as too simplistic because the requirements on phase stability can be reduced by invoking error correction ${ }^{39}$; however, this comes at the cost of increased overhead ${ }^{18}$. For qubit levels with a transition frequency in the microwave regime, local oscillators of the required phase stability exist, but the optical path lengths between the driving lasers and each of the interaction regions must be stable at the nanometre level over the course of the computation ${ }^{13}$, a daunting task for a $10 \mathrm{~cm}$ QCCD device.

On the other hand, as the logic levels of a DFS-encoded qubit are degenerate, we do not need phase synchronization at all to perform a logic operation within the DFS. Operations in the DFS are also independent of the optical path lengths of the driving lasers, because the phases $\Delta \phi_{12}, \Delta \phi_{34}$ in equations (2) and (5) depend only on the distance between the two ions comprising a logical qubit. The universal gate-set constructed above allows us to perform highly parallel computations in the DFS without synchronization between gates separated in time or space. Similar considerations would apply to other quantum computing architectures.

doi:10.1038/nature00784

\footnotetext{
1. Shor, P. W. in Proc. 35th Annu. Symp. on the Foundations of Computer Science (ed. Goldwasser, S.) 124-134 (IEEE Computer Society, Los Alamitos, 1994).
}

2. Grover, L. K. Quantum mechanics helps in searching for a needle in a haystack. Phys. Rev. Lett. 79,
325-328 (1997).

3. Cirac, J. I. \& Zoller, P. Quantum computations with cold trapped ions. Phys. Rev. Lett. 74, 4091-4094 (1995).

4. DiVincenzo, D. P. in Scalable Quantum Computers (eds Braunstein, S. L. \& Lo, H. K.) 1-14 (WileyVCH, Berlin, 2001).

5. Monroe, C. et al. Resolved-sideband Raman cooling of a bound atom to the 3D zero-point energy. Phys. Rev. Lett. 75, 4011-4014 (1995).

6. King, B. E. et al. Cooling the collective motion of trapped ions to initialize a quantum register. Phys. Rev. Lett. 81, 1525-1528 (1998).

7. Roos, C. et al. Quantum state engineering on an optical transition and decoherence in a Paul trap. Phys. Rev. Lett. 83, 4713-4716 (1999).

8. Monroe, C., Meekhof, D. M., King, B. E., Itano, W. M. \& Wineland, D. J. Demonstration of a fundamental quantum logic gate. Phys. Rev. Lett. 75, 4714-4717 (1995).

9. Sackett, C. A. et al. Experimental entanglement of four particles. Nature 404, 256-259 (2000).

10. Nägerl, H. C. et al. Laser addressing of individual ions in a linear ion trap. Phys. Rev. A 60, 145-148 (1999).

11. Blatt, R. \& Zoller, P. Quantum jumps in atomic systems. Eur. J. Phys. 9, 250-256 (1988).

12. Rowe, M. A. et al. Experimental violation of a Bell's inequality with efficient detection. Nature 409, 791-794 (2001)

13. Wineland, D. J. et al. Experimental issues in coherent quantum-state manipulation of trapped atomic ions. J. Res. NIST 103, 259-328 (1998).

14. Hughes, R. J., James, D. F. V., Knill, E. H., Laflamme, R. \& Petschek, A. G. Decoherence bounds on quantum computation with trapped ions. Phys. Rev. Lett. 77, 3240-3243 (1996).

15. Enzer, D. G. et al. in Experimental Implementation of Quantum Computation '01 (ed. Clark, R.) (Rinton, Princeton, 2001).

16. Pellizzari, T., Gardiner, S. A., Cirac, J. I. \& Zoller, P. Decoherence, continuous observation, and quantum computing: A cavity QED model. Phys. Rev. Lett. 75, 3788-3791 (1995).

17. DeVoe, R. G. Elliptical ion traps and trap arrays for quantum computation. Phys. Rev. A 58, 910-914 (1998).

18. Steane, A. M. \& Lucas, D. M. Quantum computing with trapped ions, atoms and light. Fortsch. Phys. 48, 839-858 (2000).

19. Cirac, J. I. \& Zoller, P. A scalable quantum computer with ions in an array of microtraps. Nature 404, 579-581 (2000).

20. Steane, A. et al. Speed of ion-trap quantum-information processors. Phys. Rev. A 62, 042305 (2000).

21. Sørensen, A. \& Mølmer, K. Entanglement and quantum computation with ions in thermal motion. Phys. Rev. A 62, 022311 (2000).

22. Paul, W. Electromagnetic traps for charged and neutral particles. Rev. Mod. Phys. 62, 531-540 (1990).

23. Turchette, Q. A. et al. Heating of trapped ions from the quantum ground state. Phys. Rev. A 61, 063418 (2000).

24. Kielpinski, D.. Entanglement and Decoherence in a Trapped-ion Quantum Register. Thesis, Univ. Colorado (2001); available at 〈http://www.boulder.nist.gov/timefreq/ion/qucomp/papers.htm〉.

25. Rowe, M. A. et al. Transport of quantum states and separation of ions in a dual RF ion trap. Preprint quant-ph/0205094 at $\langle$ http://xxx.lanl.gov〉 (2002).

26. Guthöhrlein, G. R., Keller, M., Hayasaka, K., Lange, W. \& Walther, H. A single ion as a nanoscopic probe of an optical field. Nature 414, 49-51 (2001).

27. Kielpinski, D. et al. Sympathetic cooling of trapped ions for quantum logic. Phys. Rev. A 61, 032310 (2000).

28. Morigi, G. \& Walther, H. Two-species Coulomb chains for quantum information. Eur. Phys. J. D 13, 261-269 (2001).

29. Larson, D. J., Bergquist, J. C., Bollinger, J. J., Itano, W. M. \& Wineland, D. J. Sympathetic cooling of trapped ions: A laser-cooled two-species nonneutral ion plasma. Phys. Rev. Lett. 57, 70-73 (1986).

30. Rohde, H. et al. Sympathetic ground-state cooling and coherent manipulation with two-ion crystals. J. Opt. B 3, S34-S41 (2001).

31. Blinov, B. B. et al. Sympathetic cooling of trapped $\mathrm{Cd}^{+}$isotopes. Preprint quant-ph/0112084 at $\langle$ http://xxx.lanl.gov $(2001)$.

32. Lidar, D. A., Chuang, I. L. \& Whaley, K. B. Decoherence-free subspaces for quantum computation. Phys. Rev. Lett. 81, 2594-2597 (1998).

33. Duan, L. M. \& Guo, G. C. Reducing decoherence in quantum-computer memory with all quantum bits coupling to the same environment. Phys. Rev. A 57, 737-741 (1998).

34. Kielpinski, D. et al. A decoherence-free quantum memory using trapped ions. Science 291, 1013-1015 (2001).

35. Sørensen, A. \& Mølmer, K. Quantum computation with ions in thermal motion. Phys. Rev. Lett. 82, 1971-1974 (1999).

36. van Enk, S. J. The physical meaning of phase and its importance for quantum teleportation. J. Mod. Opt. 48, 2049-2054 (2001).

37. Steane, A. The ion-trap quantum information processor. Appl. Phys. B 64, 623-643 (1997).

38. Young, B. C., Cruz, F. C., Itano, W. M. \& Bergquist, J. C. Visible lasers with subhertz linewidths. Phys. Rev. Lett. 82, 3799-3802 (1999).

39. Nielsen, M. A. \& Chuang, I. L. Quantum Computation and Quantum Information 425-493 (Cambridge Univ. Press, Cambridge, 2000).

\section{Acknowledgements}

We acknowledge the experimental contributions of the NIST Ion Storage group, and also J. Beall for assistance with microfabrication. We thank D. Leibfried and M.A. Rowe for comments on the manuscript. D.K. and D.J.W. were supported by the US National Security Agency (NSA), Advanced Research and Development Activity (ARDA) and the Office of Naval Research. C.M. was supported by the US NSA, ARDA and the National Science Foundation ITR programme.

Correspondence and requests for materials should be addressed to D.K.

(e-mail: utonium@mit.edu). 